\title{
Knockdown of hMex-3A by small RNA interference suppresses cell proliferation and migration in human gastric cancer cells
}

\author{
HONG JIANG $^{1,2}$, XUEMEI ZHANG ${ }^{1}$, JINHONG LUO ${ }^{1}$, CHUNYAN DONG $^{1}$, JUNLI XUE ${ }^{1}$, \\ WEI WEI ${ }^{1},{\text { JINGDE } \mathrm{CHEN}^{1} \text {, JUN ZHOU }}^{1}$, YONG GAO ${ }^{1}$ and CHANGQING YANG ${ }^{2}$ \\ ${ }^{1}$ Department of Oncology, Shanghai East Hospital of Tongji University; ${ }^{2}$ Department of Gastroenterology, \\ Tongji Hospital of Tongji University, Shanghai, P.R. China
}

Received January 22, 2012; Accepted April 20, 2012

DOI: $10.3892 / \mathrm{mmr} .2012 .943$

\begin{abstract}
RNA-binding proteins (RBPs) play essential roles in RNA metabolism, regulating RNA splicing, transport, surveillance, decay and translation. The aberrant expression of RBPs leads to gene expression alteration and frequently causes various diseases, such as cancer. In this study, we are the first to provide evidence that hMex-3A, a RBP that belongs to the human Mex-3 family with two K-homology RNA-binding domains, is involved in the regulation of tumorigenesis. We show that the silencing of hMex-3A by small interference RNA effectively inhibits cell proliferation in SNU-16 and AGS gastric cancer cells. Flow cytometry analysis confirmed this effect on SNU-16 cell growth and indicated that hMex-3A may function in the G1/M phase. Notably, hMex-3A knockdown also reduced the colony formation ability of SNU-16 and AGS cells in soft agar, implying that $\mathrm{hMex}-3 \mathrm{~A}$ is required for cell transformation. Furthermore, the hMex-3A knockdown markedly affected the migratory ability of BCG-823 cells by Transwell chamber and wound healing assays. Clinical relevance analysis using 22 paired gastric cancer specimens by quantitative real-time PCR showed that hMex-3A was significantly upregulated $(63.6 \%)$ in cancer tissues compared with matched adjacent non-cancerous tissues. Taken together, these results suggest that hMex-3A functions as an oncogene candidate in the development and metastasis of gastric cancer; thus it may serve as a potential target for the therapy of tumors.
\end{abstract}

\section{Introduction}

The regulation of mRNA post-transcriptional processes plays a central role in the control of eukaryotic gene expression.

Correspondence to: Dr Changqing Yang, Department of Gastroenterology, Tongji Hospital of Tongji University, 389 Xincun Road, Shanghai 200065, P.R. China

E-mail: cqyang@mail.tongji.edu.cn

Dr Yong Gao, Department of Oncology, Shanghai East Hospital of Tongji University, 150 Jimo Road, Shanghai 200120, P.R. China

E-mail: gaoyon@hotmail.com

Key words: hMex-3A, RNA-binding protein, cell proliferation, cell migration, gastric cancer
RNA-binding proteins (RBPs) are key players in the determination of mRNA fate (1). Certain RBPs recognize common mRNA features, such as the $5^{\prime}$ cap or the $3^{\prime}$ poly (A) tail, but the majority of RBPs contain RNA-binding domains (RBDs) for recognition of secondary structures or specific sequence motifs. More than 40 RBDs have been identified thus far, such as well-characterized RNA recognition motifs (RRMs), the K-homology (KH) domain, double-stranded RNA binding motif (dsRBM), the Arg-Gly-Gly (RGG) box, DEAD/DEAH box and Piwi/Argonaute/Zwille (PAZ) domains (2). The aberrant expression of RBPs, which affect and alter many steps of RNA metabolism, are implicated in the development of various diseases, including cancer. In humans, more than 500 RBPs are known, but only a few have been identified to exert an oncogenic or tumor-suppressive function (3). These RBPs include Src-associated in mitosis, $68 \mathrm{kDa}$ (SAM68), $\beta$-catenin, serine/arginine-rich splicing factor 1 (SRSF1), KH-type splicing regulatory protein (KSRP) and human antigen $\mathrm{R}$ (HuR) (4-8). The role of other RBPs in tumorigenesis remains to be elucidated.

Human Mex-3 is a novel family of evolutionarily conserved RBPs (hMex-3A to 3D) with two KH RBDs (9). They share the highest identity with Caenorhabditis elegans Mex-3, which is involved in the establishment of anterior-posterior embryonic asymmetry and in the maintenance of germline pluripotency. $\mathrm{hMex}-3 \mathrm{D}$ is ubiquitously expressed in various tissues; however, the other three hMex-3 proteins are expressed at varying levels in different tissues. All four proteins predominantly accumulate in the cytoplasm, and shuttle between the cytoplasm and the nucleus via the CRM1-dependent export pathway (10). A previous report has demonstrated that hMex-3A and $3 \mathrm{~B}$ are two novel components of processing bodies ( $\mathrm{P}$ bodies), in which they co-localize and interact with the hDcpla decapping factor (9). P bodies are the sites of mRNA decay and storage of non-translated transcripts, indicating that hMex-3 may be involved in the regulation of a variety of mRNA decay. In addition, hMex-3A and 3B are also associated with Argonaute (Ago) proteins. Ago1 and Ago2, the key components of the RNA-induced silencing complex, have been implicated in tumor development (11). However, it remains unknown whether the hMex-3 proteins play a role in human tumorigenesis.

Gastric cancer is one of the most common malignancies worldwide, particularly in Eastern Asian countries (12). In this 
study, we focused on hMex-3A, one of the hMex-3 proteins, and explored its role in gastric cancer development and metastasis. The results revealed that the hMex-3A knockdown inhibited cell proliferation, reduced the ability of cell transformation and suppressed cell migration. In addition, it was also found that the expression of hMex-3A was upregulated in gastric cancer tissues compared with matched adjacent non-cancerous tissues. Thus, these data provide new insight into the role of hMex-3 proteins in tumorigenesis, as well as a novel promising therapeutic target for gastric cancer.

\section{Materials and methods}

Tissue specimens and cell culture. Paired gastric cancer and adjacent paracancer tissues from 22 patients who underwent surgical resection were obtained with informed consent. The use of these tissue materials for research was approved by the Ethics Committee of the Shanghai East Hospital. Clinical and pathological information was extracted from the medical charts and pathology reports of these patients. The diagnoses of these gastric cancer samples were verified by pathologists. The three gastric cancer cell lines, SNU-16, AGS and BCG823, were obtained from the American Type Culture Collection (Manassas, VA, USA). Cells were grown in Dulbecco's modified Eagle's medium (DMEM) supplemented with $10 \%$ fetal bovine serum (FBS; Gibco) and antibiotics $(50 \mathrm{U} / \mathrm{ml}$ penicillin and $50 \mu \mathrm{g} / \mathrm{ml}$ streptomycin) (Gibco) at $37^{\circ} \mathrm{C}$ in a $5 \%$ $\mathrm{CO}_{2}$-humidified incubator.

RNA extraction and quantitative real-time PCR. Total RNA was extracted from the tissue samples or the cultured cell lines using TRIzol reagent (Invitrogen) and reverse-transcribed into cDNA using the M-MLV reverse transcriptase kit (Promega). The following primers were used to amplify the Mex-3A and $\beta$-actin genes: Mex-3A forward, 5'-ATCGTGGGCAGG CAAGGCT-3' and reverse, 5'-GCTGCTGAGATGATTT CCC-3'; $\beta$-actin forward, 5'-AGAGCCTCGCCTTTGCCGA TCC-3' and reverse, 5'-CTGGGCCTCGTCGCCCACATA-3'. Quantitative real-time PCR was performed with a Thermal Cycler Dice Real Time System (Takara) and SYBR-Green I reagent (Takara). The mRNA level of each sample was normalized to that of $\beta$-actin prior to comparative analysis using the $2^{-\Delta \mathrm{Ct}}$ method.

RNA interference and cell transfection. In order to suppress the $h M e x-3 A$ expression in gastric cancer cells, hMex-3Aspecific small interference RNAs (siRNAs) were chemically synthesized (GenePharma). The siRNA sequences used were as follows: si-2270 (sense, 5'-GUGUUUCCCUUCACU CUCUdTdT-3' and antisense, 5'-AGAGAGUGAAGGG AAACACdTdT-3'); si-5932 (sense, 5'-CUAGUGAAGAC ACGUACAAdTdT-3' and antisense, 5'-UUGUACGUGUCUU CACUAGdTdT-3'). Irrelevant nucleotides not targeting any annotated human genes were used as the negative control as follows: si-NC (sense, 5'-UUCUCCGAACGUGUCAC GUdTdT-3' and antisense, 5'-ACGUGACACGUUCGGAG AAdTdT-3'). The siRNA was subsequently transfected into the indicated cells at a final concentration of $50 \mathrm{nM}$ using Lipofectamine 2000 reagent (Invitrogen) according to the manufacturer's instructions.
Cell viability assay. Gastric cancer cells transfected with siRNAs were seeded into 96 -well plates at a density of $3 \times 10^{3}$ cells per well in $100 \mu 1$ medium. Cell viability was measured using the Cell Counting Kit-8 (Dojindo Laboratories) according to the manufacturer's instructions. Each assay was independently repeated at least three times.

Cell cycle analysis. Following transfection with siRNAs at 48 h, SNU-16 cells were harvested, fixed in cold 70\% ethanol, washed and rehydrated in PBS. DNA staining was achieved by treating the cells with RNase A $(10 \mathrm{mg} / \mathrm{ml})$ for $30 \mathrm{~min}$ and with propidium iodide $(10 \mu \mathrm{g} / \mathrm{ml})$ (Sigma) for $5 \mathrm{~min}$. Flow cytometry analysis was performed using a FACSCalibur instrument (Becton-Dickinson, Mountain View, CA, USA) and CellQuest software (Becton-Dickinson).

Soft agar colony formation assay. hMex-3A knockdown cells or control cells were suspended in medium containing $0.4 \%$ agar and overlaid on 1\% agar in 24-well plates (500 cells/well), respectively. After two-three weeks, colonies were counted and photographed. The results were expressed as the means \pm SD of triplicate counts within the same experiment.

Cell migration assay. Cell migration assays were performed using 24-well transwells (8- $\mu \mathrm{m}$ pore size, BD Biosciences) according to the manufacturer's instructions. Briefly, BCG-823 cells were trypsinized and washed three times in DMEM without FBS. A total of $5 \times 10^{4}$ cells was then suspended in $500 \mu 1$ DMEM without FBS and added to the upper chamber, while $750 \mu \mathrm{l}$ DMEM containing 10\% FBS was placed in the lower chamber. The cells were incubated for 24 to $48 \mathrm{~h}$ at $37^{\circ} \mathrm{C}$ in a $5 \% \mathrm{CO}_{2}$-humidified incubator. The non-migrating cells were removed with cotton swabs, and the migrated cells were fixed in $4 \%$ paraformaldehyde and stained with $0.5 \%$ crystal violet. Cells in at least five random microscopic fields (magnification, $\mathrm{x} 200$ ) were counted and photographed. All experiments were performed in duplicate and repeated three times.

Wound-healing assay. The cells transfected with siRNAs were grown to $90-100 \%$ confluence in six-well plates. Cell monolayers were wounded with a sterile pipette tip and then rinsed with PBS to remove cellular debris. The phase contrast images of the wounds were recorded at $37^{\circ} \mathrm{C}$ for incubations of 0,24 and $48 \mathrm{~h}$, and three separate experiments were performed.

Statistical analysis. All statistical analyses were evaluated using the Student's t-test. $\mathrm{P}<0.05$ was considered to indicate a statistically significant difference.

\section{Results}

Silencing of hMex-3A by RNA interference inhibits gastric cancer cell growth. To assess whether hMex-3A has an effect on cell growth, RNA interference against hMex-3A was carried out in the three gastric cancer cell lines, SNU-16, AGS and BCG-823. As shown in Fig. 1A, the chemically synthesized siRNAs, si-2270 and si-5932, exhibited an efficient knockdown of hMex-3A relative to the control, si-NC. Cell viability assays using the Cell Counting Kit- 8 indicated that cells in which hMex-3A was knocked down grew more slowly than in the 
A
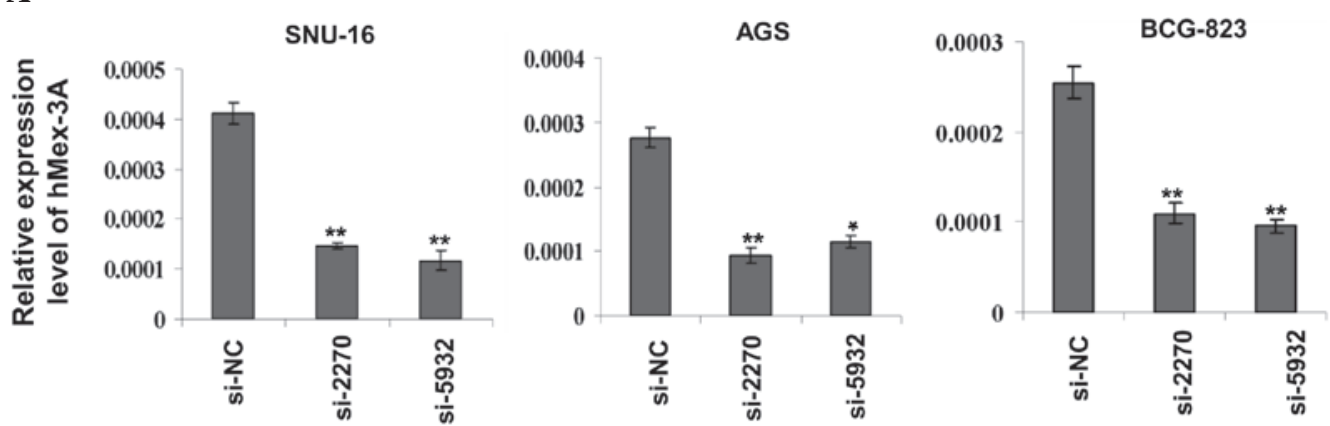

B
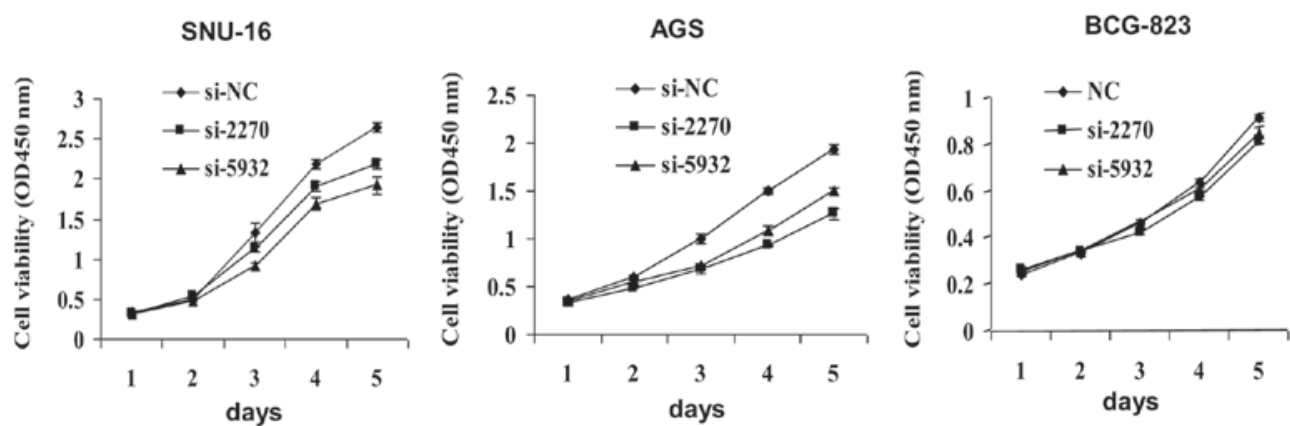

C
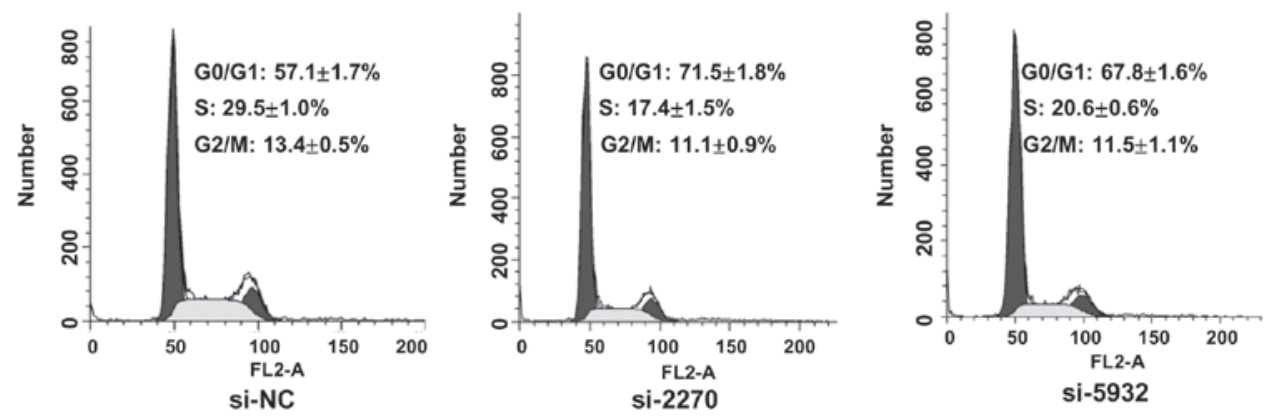

Figure 1. Effect of hMex-3A knockdown on cell growth and the cell cycle. (A) Synthesized si-2270 and si-5932 knocked down the endogenous hMex-3A in SNU-16, AGS and BCG-823 cells, as indicated by quantitative real-time PCR. (B) hMex-3A knockdown suppressed the cell growth of SNU-16 and AGS cells, where si-NC was used as the control. hMex-3A knockdown only had a slight effect on BCG-823 cells. Data are represented as the means \pm SD of three independent experiments. ${ }^{*} \mathrm{P}<0.05$ and ${ }^{* *} \mathrm{P}<0.01$. (C) SNU-16 cells were analyzed by flow cytometry after transfection with si-2270 or si-5932. The cells transfected with si-NC were used as the controls.

controls (shown for SNU-16 and AGS cells) (Fig. 1B). However, there was no marked difference observed in the BCG-823 cells, implying that the effect of hMex-3A on cell growth is dependent on cell type. The effect of hMex-3A on the cell cycle of SNU-16 cells was then evaluated by flow cytometry. As observed in Fig. 1C, the percentages of cells in the $\mathrm{S}$ phase were $29.5 \%$ in those transfected with si-NC, $17.4 \%$ in those transfected with si-2270 and $20.6 \%$ in those transfected with si-5932. These results suggest that the silencing of hMex-3A inhibits cell proliferation by lengthening the cell cycle.

Knockdown of hMex-3A affects anchorage-independent growth of SNU-16 and AGS cells. An important hallmark of cellular transformation is cell anchorage-independent growth. Normal cells often require a solid substratum on which to proliferate, whereas malignant cells gain the ability to grow regardless of their attachment status. Previous studies have reported that SNU-16 and AGS cells have such an ability of growth $(13,14)$. To examine whether hMex-3A is capable of contributing to anchorage-independent growth, a soft agar colony formation assay was performed in these two cell lines.
Compared to those cells transfected with si-NC, the cells transfected with si-2270 and si-5932 formed smaller and fewer colonies in the two cell lines (Fig. 2), indicating that hMex-3A knockdown reduced the ability of anchorage-independent growth of gastric cancer cells.

Knockdown of hMex-3A suppresses cell migration of $B C G-823$ cells. In order to further characterize the function of hMex-3A in cancer progression, a Transwell chamber assay was performed for cell migration in BCG-823 cells. Since the knockdown of hMex-3A in BCG-823 cells only had a slight effect on cell growth, the possibility that the impact on cell migration was due to cell proliferation was excluded. After $24 \mathrm{~h}$ of suspending the cells in the upper chamber, it was found that the cells transiently transfected with si-2270 and si-5932 migrated to the lower chamber less than those transiently transfected with si-NC (Fig. 3A). Furthermore, a wound healing assay was also performed in BGC823 cells. Consistent with the results from the Transwell assays, hMex-3A knockdown cells displayed a significant decrease in the cell migration ability compared with the control (Fig. 3B). 

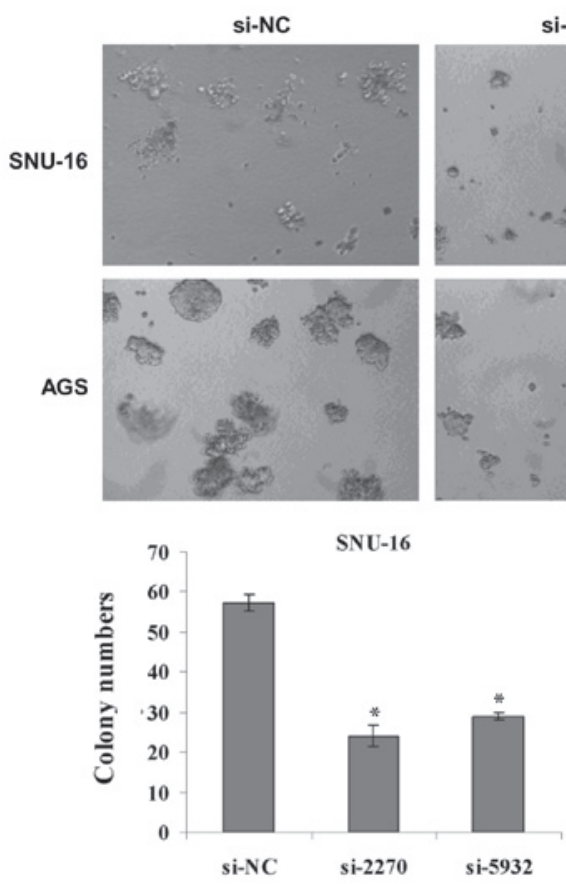

si-2270
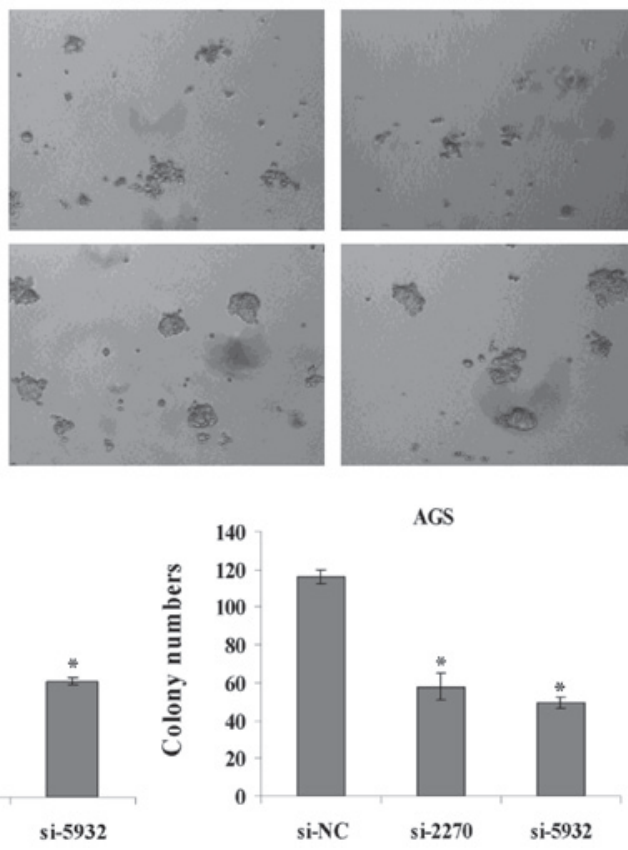

si-5932

AGS
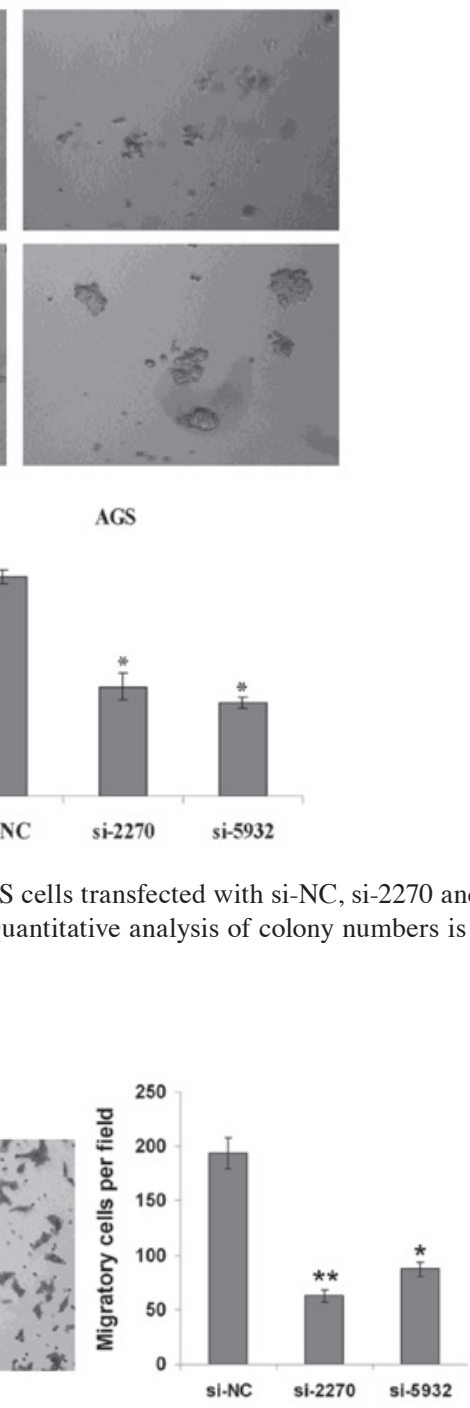

B

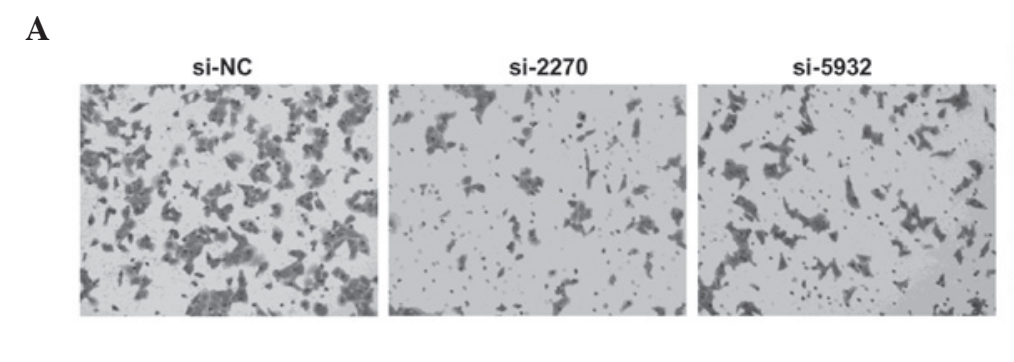

Figure 2. Knockdown of hMex-3A affects cell anchorage-independent growth. SNU-16 and AGS cells transfected with si-NC, si-2270 and si-5932 were used for the soft agar colony formation assay. Representative cell colonies in soft agar are shown. Quantitative analysis of colony numbers is shown in the lower panel. Values are the means \pm SD of at least three independent experiments. ${ }^{*} \mathrm{P}<0.05$.

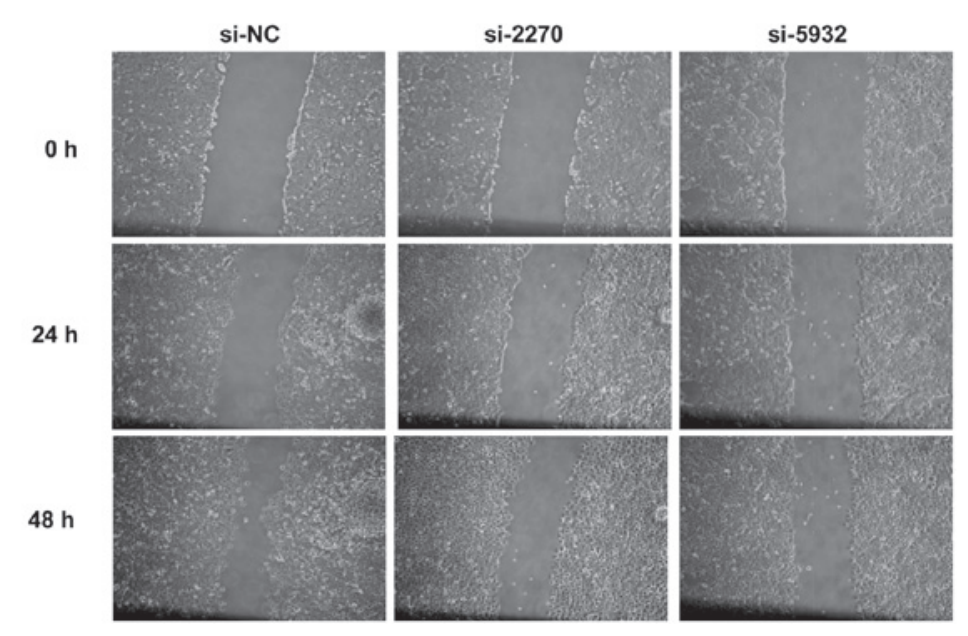

Figure 3. Downregulation of hMex-3A suppresses cell migration. (A) Transfected BCG-823 cells with indicated siRNAs seeded into the upper compartment of the Transwell culture chambers and incubated for $24 \mathrm{~h}$. Migrated cells were visualized by crystal violet staining under microscope observation (magnification, x200). " $\mathrm{P}<0.05$ and ${ }^{* *} \mathrm{P}<0.01$ compared with the controls. (B) Wound healing assay was performed on monolayers of BCG-823 cells transfected with indicated siRNAs. Representative images were taken at $0,24 \mathrm{~h}$ and $48 \mathrm{~h}$ after wounds were made.

Taken together, these data demonstrate that a decrease in the hMex-3A level markedly influences cell migration, implying that hMex-3A contributes to the metastasis of gastric cancer.
hMex-3A is frequently upregulated in gastric cancer tissues. To investigate the clinical relevance of $\mathrm{hMex}-3 \mathrm{~A}$ in gastric cancer, the mRNA levels of hMex-3A in 22 pairs of human 
A

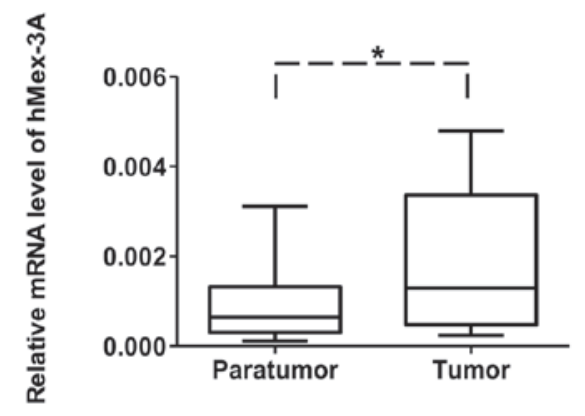

B

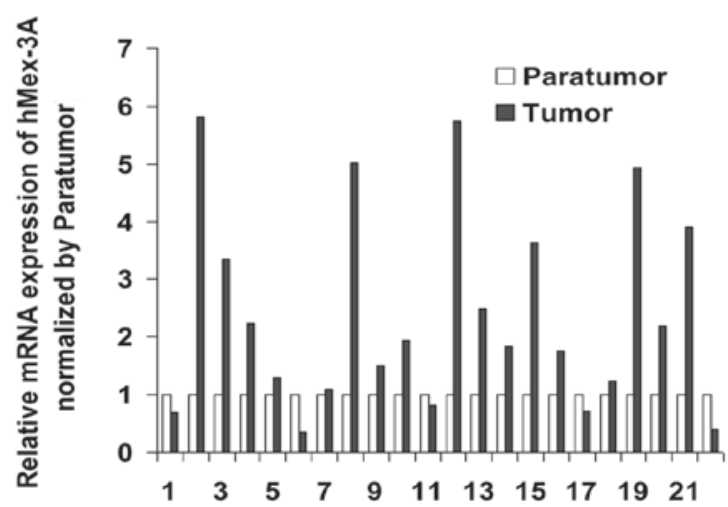

Figure 4. Expression pattern of hMex-3A in gastric cancer specimens. (A) The transcript level of hMex-3A was measured in 22 paired gastric cancer specimens by quantitative real-time PCR. Relative transcript levels were normalized by the $\Delta$ Ct-method using $\beta$-actin as an internal reference. The line within each column represents the median relative value; the upper and lower edges of each box represent the 75th and 25th percentile, respectively; the upper and lower bars indicate the highest and lowest values detected, respectively. ${ }^{*} \mathrm{P}<0.05$. (B). The relative transcript level of hMex-3A in 22 paired specimens was respectively indicated. Each pair consisted of tumor tissue and paratumor tissue from the same patient.

gastric cancer samples were measured by quantitative realtime PCR. The resulting data revealed that hMex-3A was significantly upregulated in tumor tissues in comparison with adjacent non-tumorous tissues (Fig. 4A). Among the samples, 14 of $22(63.6 \%)$ displayed at least a 1.5 -fold increase in tumor tissues compared to paratumor tissues (Fig. 4B). The expression pattern of hMex-3A in clinical samples suggests its involvement in the pathogenesis of gastric cancer.

\section{Discussion}

Human Mex-3A proteins, including hMex-3A, -3B, -3C and $-3 \mathrm{D}$, are located on distinct chromosomes at $1 q 22,15 q 25.2$, $18 q 21.1$ and $19 p 13.3$, respectively (9). All the four proteins contain two highly conserved KH domains specifically for RNA binding and a RING finger domain in their carboxyterminal region probably involved in mediating protein-protein interactions (15-17). The similarity in structure of the hMex-3 proteins implies that they may share certain similar biological functions. In the present study, one of the four proteins, hMex$3 \mathrm{~A}$, was selected to investigate its function in gastric cancer development and progression for the first time. The final data demonstrated that a reduction in the hMex-3A expression level led to a suppression of cell growth in an anchorage-dependent and -independent manner. Furthermore, the silencing of $\mathrm{hMex}-3 \mathrm{~A}$ also resulted in a decrease in the migratory ability of gastric cancer cells. These findings shed light on the role of hMex-3 proteins, particularly for hMex-3A in tumorigenesis.

In recent years, RNA interference has been employed as a mature and powerful strategy for exploring gene functions by downregulating the expression of targeted genes. We applied this technique to specifically silence hMex-3A expression in the gastric cancer cell lines, SNU-16, AGS and BCG-823. Notably, the significant inhibitory effect on cell growth was present in SNU-16 and AGS cells, but not in BCG-823 cells, indicating that the effect of hMex-3A on cell growth is dependent on cell type. More importantly, flow cytometry analysis confirmed the inhibitory effect on the cell growth of SNU-16 cells. These data imply that hMex-3A may be essential for maintaining efficient cell division in gastric cancer cells. Moreover, we also show that the hMex-3A expression level severely influences cell growth ability in soft agar. This ability of cell growth in soft agar is assumed to closely reflect the ability of cell transformation in in vivo carcinogenesis. Another hallmark of tumors is metastasis. To date, tumor recurrence and metastasis remain the main obstacles in treatment. The results of cell metastasis assays revealed that hMex-3A may be involved in this process. In addition, the role of hMex-3A involvement in the gastric cancer development was supported by clinical data. Quantitative real-time PCR results indicated that the transcription level of hMex-3A was significantly upregulated in tumor tissues compared to adjacent non-tumorous tissues; however, this upregulation of hMex-3A did not statistically correlate with gender, age, tumor size or metastasis. One possible reason for this may be that we did not have not enough clinical samples for statistical analysis.

hMex-3 proteins were initially identified in a screening for genes with similarity to the Caenorhabditis elegans Mex-3. In the nematode, the Mex-3 protein acts in the cytoplasm during the early cleavage stages of the embryonic cell and is localized in P-granules (18), which are cytoplasmic structures containing RNA and RBPs playing a role in mRNA processing or packaging. By contrast, hMex-3 proteins are mostly distributed in the nuclear-perinuclear cell compartment and may play a similar role in RNA metabolism as RBPs. Deregulation of RBP expression or activity has been reported in a number of malignancies $(4,19)$. RBPs are also key components in the determination of microRNA (miRNA) function, as they control different stages of miRNA biogenesis and their localization, degradation and activity. The global downregulation of miRNA expression is an emerging feature in cancer, and the specific deregulation of certain miRNAs has been observed in specific tumor types $(20,21)$. Most notably, hMex-3A has been reported to be associated with Ago proteins. Ago proteins are components of the RNA-induced silencing complex and are crucial effectors of RNA silencing $(22,23)$. Recent studies have revealed that Ago1 and Ago2 play an important oncogenic role in breast and colon cancer. These insights uncover a probable mechanism for hMex-3A in RNA regulation, which could have relevance for cancer development and progression. Taken 
together, tumorigenesis is a complicated process and the exact mechanism by which hMex-3A contributes to tumorigenesis should be explored in the future.

In conclusion, our present study is the first to show that the knockdown of hMex-3A using RNA interference can effectively inhibit cell proliferation and migration in gastric cancer cells. These results further indicate that hMex-3A may serve as a potential target for the therapy of gastric cancer, although additional studies in vivo are necessary.

\section{Acknowledgements}

This study was supported by grants from the National Natural Science Foundation of China (81070343) and Shanghai Excellent Academic leaders Program (08xD14045).

\section{References}

1. Kim MY, Hur J and Jeong S: Emerging roles of RNA and RNA-binding protein network in cancer cells. BMB Rep 42: 125-130, 2009.

2. Lunde BM, Moore C and Varani G: RNA-binding proteins: modular design for efficient function. Nat Rev Mol Cell Biol 8: $479-490,2007$

3. Lukong KE, Chang KW, Khandjian EW and Richard S: RNA-binding proteins in human genetic disease. Trends Genet 24: 416-425, 2008

4. Bielli P, Busa R, Paronetto MP and Sette C: The RNA-binding protein Sam68 is a multifunctional player in human cancer Endocr Relat Cancer 18: R91-R102, 2011.

5. Shultz JC, Goehe RW, Murudkar CS, et al: SRSF1 regulates the alternative splicing of caspase 9 via a novel intronic splicing enhancer affecting the chemotherapeutic sensitivity of non-small cell lung cancer cells. Mol Cancer Res 9: 889-900, 2011.

6. Denkert C, Koch I, von Keyserlingk N, et al: Expression of the ELAV-like protein HuR in human colon cancer: association with tumor stage and cyclooxygenase-2. Mod Pathol 19: 1261-1269, 2006.

7. Gherzi R, Trabucchi M, Ponassi M, et al: The RNA-binding protein KSRP promotes decay of beta-catenin mRNA and is inactivated by PI3K-AKT signaling. PLoS Biol 5: e5, 2006.

8. Lee HK and Jeong S: Beta-catenin stabilizes cyclooxygenase-2 mRNA by interacting with AU-rich elements of 3'-UTR. Nucleic Acids Res 34: 5705-5714, 2006.
9. Buchet-Poyau K, Courchet J, Le Hir H, et al: Identification and characterization of human Mex-3 proteins, a novel family of evolutionarily conserved RNA-binding proteins differentially localized to processing bodies. Nucleic Acids Res 35: 1289-1300, 2007.

10. Fornerod M, Ohno M, Yoshida M and Mattaj IW: CRM1 is an export receptor for leucine-rich nuclear export signals. Cell 90: 1051-1060, 1997.

11. Parisi C, Giorgi C, Batassa EM, et al: Ago1 and Ago2 differentially affect cell proliferation, motility and apoptosis when overexpressed in SH-SY5Y neuroblastoma cells. FEBS Lett 585: 2965-2971, 2011.

12. Alberts SR, Cervantes A and van de Velde CJ: Gastric cancer: epidemiology, pathology and treatment. Ann Oncol 14 Suppl 2: ii31-36, 2003

13. Kim YB, Lee SY, Ye SK and Lee JW: Epigenetic regulation of integrin-linked kinase expression depending on adhesion of gastric carcinoma cells. Am J Physiol Cell Physiol 292: C857-866, 2007

14. Xu X, Li W, Fan X, et al: Identification and characterization of a novel p42.3 gene as tumor-specific and mitosis phase-dependent expression in gastric cancer. Oncogene 26: 7371-7379, 2007.

15. Joazeiro CA and Weissman AM: RING finger proteins: mediators of ubiquitin ligase activity. Cell 102: 549-552, 2000.

16. Fang S, Lorick KL, Jensen JP and Weissman AM: RING finger ubiquitin protein ligases: implications for tumorigenesis, metastasis and for molecular targets in cancer. Semin Cancer Biol 13: 5-14, 2003.

17. Draper BW, Mello CC, Bowerman B, Hardin J and Priess JR: MEX-3 is a KH domain protein that regulates blastomere identity in early C. elegans embryos. Cell 87: 205-216, 1996.

18. Hunter CP and Kenyon C: Spatial and temporal controls target pal-1 blastomere-specification activity to a single blastomere lineage in C. elegans embryos. Cell 87: 217-226, 1996.

19. Gubin MM, Calaluce R, Davis JW, et al: Overexpression of the RNA binding protein HuR impairs tumor growth in triple negative breast cancer associated with deficient angiogenesis. Cell Cycle 9: 3337-3346,

20. Lu J, Getz G, Miska EA, et al: MicroRNA expression profiles classify human cancers. Nature 435: 834-838, 2005.

21. Volinia S, Calin GA, Liu CG, et al: A microRNA expression signature of human solid tumors defines cancer gene targets. Proc Natl Acad Sci USA 103: 2257-2261, 2006.

22. Janowski BA, Huffman KE, Schwartz JC, et al: Involvement of AGO1 and AGO2 in mammalian transcriptional silencing. Nat Struct Mol Biol 13: 787-792, 2006.

23. Adams BD, Claffey KP and White BA: Argonaute-2 expression is regulated by epidermal growth factor receptor and mitogenactivated protein kinase signaling and correlates with a transformed phenotype in breast cancer cells. Endocrinology 150: 14-23, 2009. 